\title{
Patients undergoing craniofacial tumour ablation surgery may benefit from having the implants placed simultaneously instead of waiting
}

\author{
Abstracted from \\ Barber AJ, Butterworth CJ, Rogers SN. \\ Systematic review of primary osseointegrated dental implants in head and neck oncology. \\ Br J Oral Maxillofac Surg 2010 \\ Address for correspondence: AJ Barber, Department of Restorative Dentistry, \\ University of Bristol Dental Hospital, Lower Maudlin Street, Bristol BS1 2LY, UK. E-mail: andrewbarber2@nhs.net
}

\section{Question: What is the effectiveness of primary insertion of dental implants in people who have head and neck cancer?}

Data sources Medline, Embase and the Cochrane Library were searched for studies and the reference lists of the full-text articles were checked for any additional studies.

Study selection Included studies were randomised clinical trials (RCT) and non-RCT, cohort studies, case-control studies, case reports, or reviews that addressed the placement of dental implants at the same time as primary oncological resection in people suffering from cancer of the head and neck (primary implant insertion); or addressed benign or malignant tumours and the placement of implants into the native maxilla, mandible and zygoma, and grafted tissue. Articles were restricted to those written in English. The title and abstracts were reviewed independently by two reviewers.

Data extraction and synthesis Data extraction was conducted independently and a qualitative synthesis of the data presented. Results Three case reports, 13 reviews, and 25 clinical studies were selected. Eight of the clinical studies referred solely to the insertion of dental implants at the time of primary oncological resection, and only two were of a prospective design.

Conclusions Published studies concerning primary dental implants were concisely summarised, so that the collected evidence base surrounding this approach to oral rehabilitation could inform head and neck cancer teams, particularly oncological surgeons, restorative dentists, and maxillofacial prosthodontists.

\section{Commentary}

The scientific foundation for decisions over whether patients benefit from having implants placed at the time of craniofacial ablative tumour surgery, or by delaying, seems rather weak. The authors of this systematic review do not provide any clear guidance in any direction, since there is apparently little or no evidence to support any conclusions.

The literature search for papers published from 1950 to March 2009 seemed quite comprehensive and covered seven different bibliographic databases. The procedure for study inclusion and exclusion appears to follow common guidelines for reporting systematic reviews, such as PRISMA (Preferred Reporting Items for Systematic Reviews and Meta-Analyses www.prisma-statement. org). For example, two reviewers independently selected the studies, assessed their qualities, and defined the clinical outcomes of interest that were the most clinically significant.

The majority of papers reporting on clinical studies of maxillofacial reconstructions using dental implants following ablative tumour surgery describe treatment protocols where implants are not placed at the time of surgery. Since the first paper that described a primary-placement approach appeared in 1989, 16 additional papers have reported patient outcomes using both primary and delayed implant placements. None of these provide guidance over superiority. Nevertheless, in one paper within this category, which was not included in this systematic review, the authors had clear reservations about a primary placement approach when applied to maxillectomies. ${ }^{1}$ The same group who described the primary placement protocol in the 1989 paper also reported in 1991 on 10 patients who had received implants placed at the time of ablative surgery. ${ }^{2}$ This paper and the subsequent seven clinical studies referred to in the review do not present findings that raise major concerns about following a primary implant placement protocol.

The authors here identified three case reports and 13 reviews that offer opinions on this subject. It is obvious that this topic does engage oncological and oral maxillofacial surgeons: of approximately 40 papers reporting on outcomes using the delayed implant placement protocol, many discuss the primary versus secondary implant placement approaches.

Two oncology teams in The Netherlands have identified multiple justifications for implementing a primary placement protocol. ${ }^{3,4}$ Convincing reasons are the avoidance of implant surgery in radiotherapy-compromised areas that are at high risk of osteonecrosis, and a more rapid rehabilitation of form, function, speech and swallowing. Avoidance of long-term use of antibiotics 
and hyperbaric oxygen therapy are also arguments in favour of a primary placement protocol.

Weighted against these considerations are the potential adverse effects of the additional approx. 15\% backscattering from titanium implants, and possible localised tissue damage if the patient needs to undergo radiotherapy. The exact limits and mechanisms of potential backscatter remain to be established, ${ }^{5}$ and it has been claimed that the doses can be minimised to clinical insignificance by using multiple radiation fields. ${ }^{6}$ Once again, the major concern is the dreaded osteonecrosis and its debilitating consequences, so that the precautionary principle tends to be applied. This is evidently not a new mindset. It is worth noting that a precautionary principle was also used in 1982 when titanium implants were first introduced. The decree at the time was to avoid radiographs until the abutment connection stage in fear of disturbing the osseo-integration process. $^{7}$ Although this practice has been abandoned we are still not confident about what is a maximum post-implant placement radiographic exposure.

A slightly different strategy to avoid possible detrimental additional backscatter generated during radiotherapy is the use of nonmetallic instead of titanium implants. At least one implant material, namely aluminium-oxide, appears in vitro to not generate any dose enhancements. ${ }^{8}$

An additional consideration when weighing up the many rational arguments presented in older literature is that the technological developments of implant surfaces over the last 2 decades allow a more rapid osseo-integration today than the first generation of turned dental implants. Whereas previously the healing process was estimated to be about 16 weeks, the general consensus today is that $3-4$ weeks suffice. ${ }^{9}$ This timeframe is within the healing period following the ablative surgery, before commencing radiotherapy.,4

A factor that deters decisions in favour of primary implant placement is the high risk of improper positioning if the surgery results in gross alteration of the maxilla-mandibular relationship. ${ }^{10}$ Likely cancer therapy variables are the tumour region, the tumour stage, the malignancy aggressiveness, the radiotherapy planning target volume, fractionation schedules, and total radioactivity dose. Other variables with a probable, but unknown, impact are location and orientation of placed implants, bone continuity and maxilla-mandibular relationships. Finally, the skills and experience of the surgical team is an often overlooked variable when addressing outcomes of surgical interventions.

A concern is that the tumour recurrence in this patient population is high. ${ }^{1,4,10,11}$ Statistically, a large proportion of primary placed implants, if placed, will never be restored because of tumour recurrence, poor implant position, and patient health and function issues following resection and reconstruction. Competing for limited resources and surgery team priorities is an indisputable consideration. A cost-effectiveness/-utility appraisal of the resources utilisation versus patient quality of life may possibly provide these answers. ${ }^{10,11}$ First, however, the necessary clinical data foundation needs to be built.

A genuine obstacle to addressing risk and prognostic factors on outcomes following primary versus secondary implant placement is that clinical centres mostly work in isolation and, because of the low incidence of this type of surgery, are not able to establish statistical evidence. Extra-oral implants are more infrequent than intra-oral placements and the seven papers reporting on this application were excluded in this systematic review. Thus, it remains unknown whether the outcomes of extra-oral and intraoral implants are comparable, as a function of primary or secondary placement, with or without subsequent radiotherapy. With regard to both intra- and extra-oral implants, international cooperative efforts are needed to determine which patients may benefit from primary implant placement and where a delayed strategy seems the most advantageous.

Asbjørn Jokstad

University of Toronto, Faculty of Dentistry, Toronto, Canada

1. Roumanas ED, Nishimura RD, Davis BK, Beumer 3rd J. Clinical evaluation of implants retaining edentulous maxillary obturator prostheses. J Prosthet Dent 1997; 77: 184-190.

2. Urken ML, Buchbinder $\mathrm{D}$, Weinberg $\mathrm{H}$, et al. Functional evaluation following microvascular oromandibular reconstruction of the oral cancer patient: a comparative study of reconstructed and nonreconstructed patients. Laryngoscope 1991; 101: 935-950.

3. Schoen PJ, Reintsema H, Raghoebar GM, Vissink A, Roodenburg JL. The use of implant retained mandibular prostheses in the oral rehabilitation of head and neck cancer patients. A review and rationale for treatment planning. Oral Oncol 2004; 40: 862-871.

4. Schepers RH, Slagter AP, Kaanders JH, van den Hoogen FJ, Merkx MA. Effect of postoperative radiotherapy on the functional result of implants placed during ablative surgery for oral cancer. Int J Oral Maxillofac Surg 2006; 35: 803-808.

5. Jegoux F, Malard O, Goyenvalle E, Aguado E, Daculsi G. Radiation effects on bone healing and reconstruction: interpretation of the literature. Oral Surg Oral Med Oral Pathol Oral Radiol Endod 2010; 109: 173-184.

6. Wang R, Pillai K, Jones PK. Dosimetric measurement of scattered radiation from dental implants in simulated head and neck radiotherapy. Int J Oral Maxillofac Implants 1998; 13: 197-203.

7. Strid KG. Radiographic procedures. In Tissue-integrated Prostheses. Osseointegration in Clinical Dentistry. Edited by Brånemark PI, Zarb GA, Albrektsson T. Chicago: Quintessence; 1985: p317.

8. Binger T, Seifert H, Blass $G$, Bormann $K H$, Rücker M. Dose inhomogeneities on surfaces of different dental implants during irradiation with high-energy photons. Dentomaxillofac Radiol 2008; 37: 149-153.

9. Raghavendra S, Wood MC, Taylor T. Early wound healing around endosseous implants: a review of the literature. Int J Oral Maxillofac Implants 2005: 20: 425-431.

10. Schoen PJ, Raghoebar GM, Bouma J, et al. Prosthodontic rehabilitation of oral function in head-neck cancer patients with dental implants placed simultaneously during ablative tumour surgery: an assessment of treatment outcomes and quality of life. Int J Oral Maxillofac Surg 2008; 37: 8-16.

11, Garrett N, Roumanas ED, Blackwell KE, et al. Efficacy of conventional and implantsupported mandibular resection prostheses: study overview and treatment outcomes. J Prosthet Dent 2006; 96: 13-24.

Evidence-Based Dentistry (2010) 11, 52-53. doi:10.1038/sj.ebd.6400723 